\title{
Development of a Novel Multi-Functional Rehabilitation Robot
}

\section{Cheng-dong WEI ${ }^{1}$, Quan-quan LIU ${ }^{1,2}$, Li-hong DUAN ${ }^{1,3}$, Tong-yang $\mathrm{SUN}^{2}$, Meng $\mathrm{LI}^{1}$, Zhan ZENG ${ }^{4}$, Chun-bao WANG ${ }^{1,2,5, \mathrm{a},{ }^{,} \text {, Qing SHI }}{ }^{6}$, Zhuo-hua LIN ${ }^{6}$, Ya-jing SHEN ${ }^{5}$, Wan-feng SHANG ${ }^{7}$ and Zheng-zhi $W U^{1, b, *}$}

\author{
${ }^{1}$ Shenzhen Institute of Geriatrics, Shenzhen, China. \\ ${ }^{2}$ School of Mechanical and Automotive Engineering, South China University of \\ Technology, Guangzhou, 510640, China \\ ${ }^{3}$ Mechanical and Biomedical Engineering, City University of Hongkong, China. \\ ${ }^{4}$ School of Electronic Engineering and Automation, \\ Guilin University of Electronic Technology, Guilin, 541004, China \\ ${ }^{5}$ Guangxi University of Science and Technology, China \\ ${ }^{6}$ Faculty of Science and Engineering, Waseda University, Japan. \\ ${ }^{7}$ Faculty of Mechanical Engineering, Chinese University of Hongkong, China. \\ achunbaowang@163.com, bszwzz001@163.com \\ ${ }^{*}$ Corresponding authors
}

Keywords: Multi-functional, Support manipulator, Rehabilitation.

\begin{abstract}
With the development of aging society, the number of hemiplegia patients grows rapidly and over $75 \%$ of survivors suffering hemiplegia need rehabilitation training. Traditional training methods need one or more physical therapists to server one patient at the same time. In order to reduce the workload of the physical therapist and to improve patient rehabilitation performance, this paper presents a novel multi-functional rehabilitation robot, which can assist hemiplegic patient for stand up/squatting training, Gravity shifting training and Walking training. The robot system consists of two Support manipulator, Screw lift mechanism, Weight balance device and a Treadmill. Different from the existing rehabilitation robot, the design philosophy of the multi-functional support robot is to mimic hand movement of physical therapist. It is flexible to fit in patients with individual differences. The design of the detailed mechanical components is illustrated. The proposed robot system can be used in multi-function exercise for hemiplegic patient.
\end{abstract}

\section{Introduction}

China in the 21 st century will be fully into the aging society, according to the record of Chinese National Bureau of Statistics, As of 2015the population over 60 years old China's elderly population has reached 220 million, the level of aging is $15 \%$. And from 2030 to 2050, the aging rate will show a linear increase in the situation. It is expected that in 2050, the total number of elderly people in China will reach 450 million, accounting for more than $30 \%$ of the total population [1]. As the aging population is growing, the aging society caused the disease situation more and more severe. Stroke is a high incidence of disease among elderly people. About $75 \%$ of survivors after stroke 
have disability, more than $40 \%$ for severely disabled. So the elderly service and rehabilitation industry in the world will pay attention [2].

For stroke sequelae, traditional rehabilitation training includes Gravity shifting training (Fig.1.a), stand up/squatting training (Fig.1.b) and Walking training(Fig.1.c) [3]. The main means of rehabilitation is based on the therapis to treat the patient as one to one, even several-to-one. Which makes the number of therapists seriously inadequate, and the rehabilitation effect is too dependent on the therapists' own technology and experience, heavy training process makes the therapists' work burden is too large. These factors have a serious impact on the patient's rehabilitation effect .

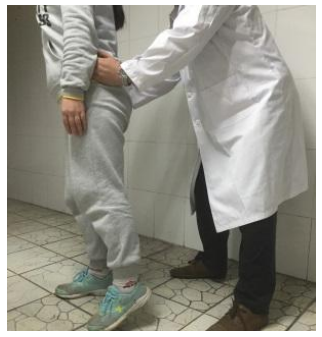

(a)

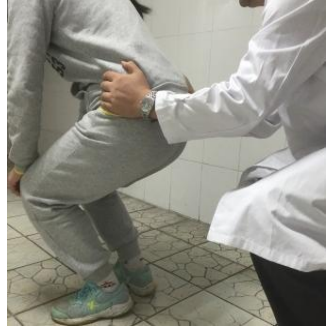

(b)

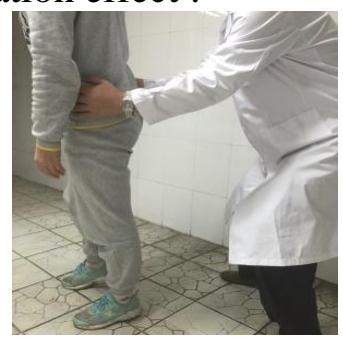

(c)

Figure 1. Traditional rehabilitation training

The existing lower limb rehabilitation robot from the functional and structural can be divided into single weight-free robot, pedal robot, bed robot, exoskeleton robot and so on. Single weight-free robot, such as Kine Assist [4] of the University of Chicago, which is shown in Fig.2.(a). Pedal robot, such as German Gait Trainer [5], which is shown in Fig.2.(b). Bed robot, such as Swiss Erigo [6], which is shown in Fig.2.(c). Exoskeleton robot, such as Japanese HAL[7], which is shown in Fig.2.(d). So we propose a multi-functional intelligent rehabilitation robot based on bionics design is proposed to simulate the rehabilitation of the therapists, to guide the patients to participate in the training and improve the rehabilitation training effect.

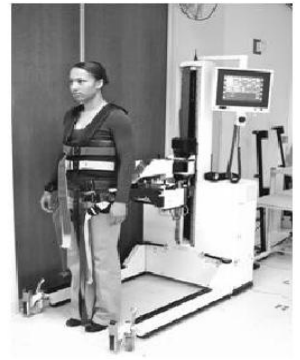

(a) Kine Assist

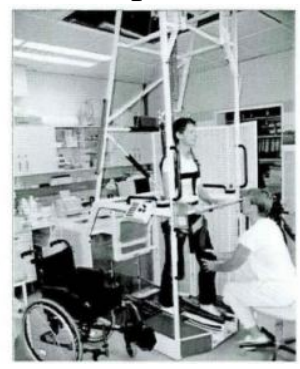

(b) Gait Trainer

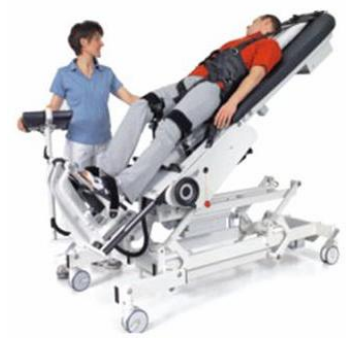

(c) Erigo

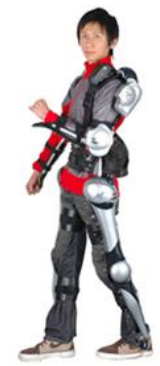

(d) HAL

Figure2. Research on lower limb robot

The existing rehabilitation robots pay more attention to the rehabilitation of patients with passive training to patient gait tend to healthy human training purpose .In this research, a novel multifunctional rehabilitation robot is proposed, its design philosophy is based on the therapist in the patient rehabilitation training process of the simulation, to guide the patients to participate in the training and improve the rehabilitation training effect. The rest of this paper is organized as follows: Section II gives an introduction of analysis requirements which are composed of the physiological requirement and the rehabilitation requirement. Section III describes the mechanism design in details. Section IV presents the conclusion and future research direction of Multi - functional rehabilitation robot. 


\section{Requirement Analysis}

\section{Physiological Requirements}

The lower limb joints of the human body are mainly composed of hip knee and ankle. Traditional rehabilitation methods mainly for hemiplegic patients with lower limb functional reconstruction training, and the most important is hip training. The hip joint is the connection between the pelvis and the caput femoris. There are 6 degrees of freedom(DOF) in the pelvic part of the normal human body, with three DOF at the pelvic left and right (along the vertical axis), pitch (coronal axis) and roll (sagittal axis) at the hip joint Coronal axis), front and rear (along the sagittal axis) and up and down (along the vertical axis) in three directions of freedom of movement. During the patient rehabilitation training, the pelvic motion can be reduced to four DOF (2R2T): Rotation about the vertical and sagittal axes, translational along the vertical axis and the crown axis. Therefore, the design of the organization must be have the degree of freedom to meet the needs of rehabilitation training. And the simplified model of human body of the therapist and the patient is shown in Fig.3.(a)-(b).

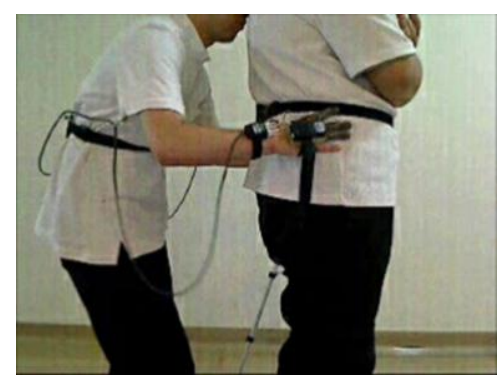

(a) Rehabilitation training

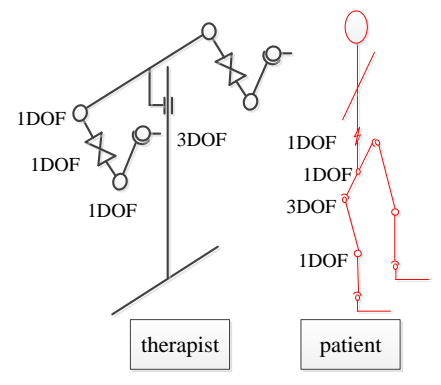

(b) Simplified body model

Figure 3. Hip joint analysis

\section{Rehabilitation Training Requirements}

According to the theory of brain plasticity and functional reorganization, function rehabilitation could realize through early training [8][9]. It means that early rehabilitation training is necessary for hemiplegic patients. For hemiplegic patients, walking is their most urgent need to restore the function, do not have the ability to walk, not only the negative impact on the patient's physical and mental, but also on the patient's family to bring a great burden of life, because they need specialized people always take care of patients. The hip is the most important joint in the walking function, which controls the lifting and moving of the legs. In the course of walking, the hip needs to be 'flexion / extension', 'adduction / abduction' and ' intorsion / extorsion ' movement, and it need for repeated high-intensity training to stimulate functional reorganization. But the cycle of fixed mode training, not only to the healer to bring heavy pressure, the patient's training effect will be affected. So this paper propose a novel rehabilitation robot which can simulate the operation of the therapist hand technology, combined with different patient differences, intelligent and parametric to develop a more conducive to the patient's treatment program.

According to the physiological requirements and rehabilitation training requirements, the multi-functional robot should achieve the following requirements: 1) At least six degree of freedom and flexible control can be achieved. 2) combining active training and passive training; 3) Safety design of the whole structure. 


\section{Hardware Configuration}

As is shown in Fig.4, robot support manipulator hold the patient's hip. In the passive training mode, to guide patients to carry out various rehabilitation training. In the active mode, to ensure that patients in the training process of physical balance. According to therapy of traditional rehabilitation training and Fig.4, the 3D model of the robot system is built, which is shown in Fig.5. The robot system is consisted of Support manipulator, Screw lift mechanism ,Weight loss deviceand Treadmill. The 3D model of robot support manipulator is shown in Fig.6, to better describe the mechanism structure, the robot support manipulator can be divided into three parts, Shoulder joint, Rotation joint and Elbow joint.

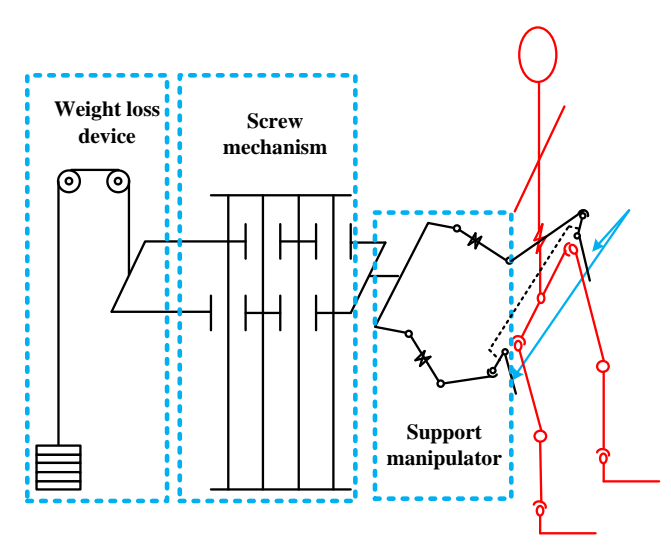

Figure4. Schematic diagram of Robot

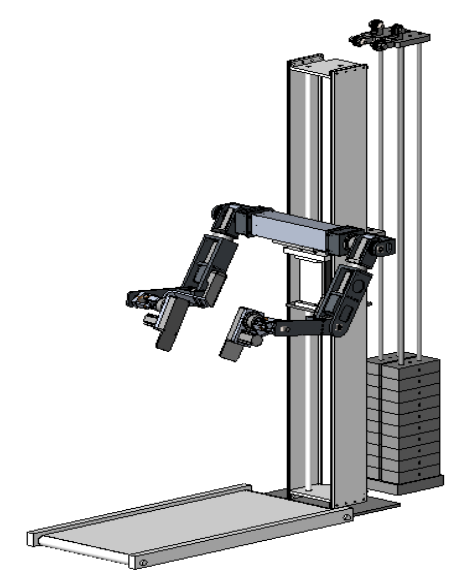

Figure5. 3D model of RrehabilitationRobot

\section{Shoulder Joint}

According to the original proposed program, rehabilitation robot design is highly matched height of $90 \%$ of the height of the body height, according to "GB10000-88 Chinese adult human size"[10] in the data can be found, $\mathrm{H}=1754 \mathrm{~mm}$. And because the hemiplegia patients with athletic ability is weak, arms support operation hand rotation speed to take $5 \mathrm{r} / \mathrm{min}$, that is $0.52 \mathrm{rad} / \mathrm{s}$; the rest of the arm joint length and weight and other data:

Upper arm length L1 $=333 \mathrm{~mm}$. Forearm length $\mathrm{L} 2=253 \mathrm{~mm}$. Weight $\mathrm{G}=71 \mathrm{~kg}$.

Arms support rehabilitation robot design weight reduction mechanism, the maximum weight loss $50 \mathrm{KG}$, so the motor bear the maximum weight of $\mathrm{G} 1=21 \mathrm{~kg}$.

$$
\begin{aligned}
& \omega=0.52 \mathrm{rad} / \mathrm{s} \\
& \mathrm{T}=\mathrm{G} 1 * \mathrm{~L}=123.06 \mathrm{Nm} \\
& \mathrm{P}=\omega \mathrm{T}=64 \mathrm{~W}
\end{aligned}
$$

Wherewrepresents speed, $\mathrm{T}$ represents the torque, $\mathrm{P}$ represents the power. 


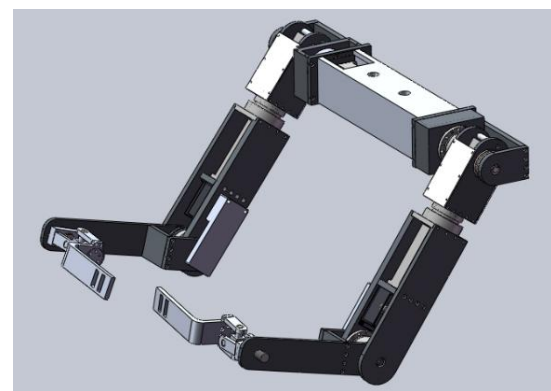

Figure6. 3D model of Support Manipulator joint

Considering the 1.5 times safety margin, the output power of motor is $96 \mathrm{~W}$. Techservo MT series brushless DC motor ST10N40P10V2-BK for 100W motor is chosen. The output torque of the motor is $0.32 \mathrm{Nm}$, and the rated speed is $3000 \mathrm{rpm}$. The transmission relationship of Shoulder joint is illustrated in Fig.7. Use the same method to calculate the rotary joint and elbow of the motor power, both of the motor power is $100 \mathrm{w}$ for Techservo MT series brushless DC motor ST10N40P10V2-BK. The transmission relationship of Rotation joint is illustrated in Fig.8. The transmission relationship of Elbow joint is illustrated in Fig.9. In order to fix the requirement, the ratio of the reducer, which are shown in Tab.1.

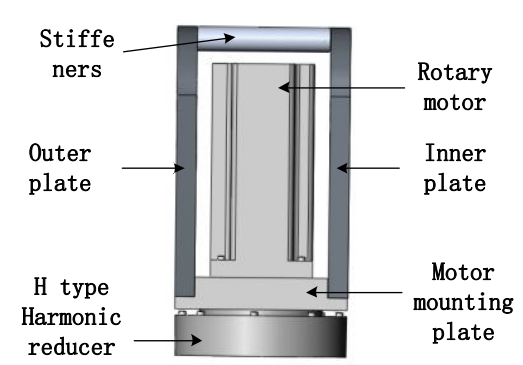

Figure8. Transimission relationship of Rotation joint

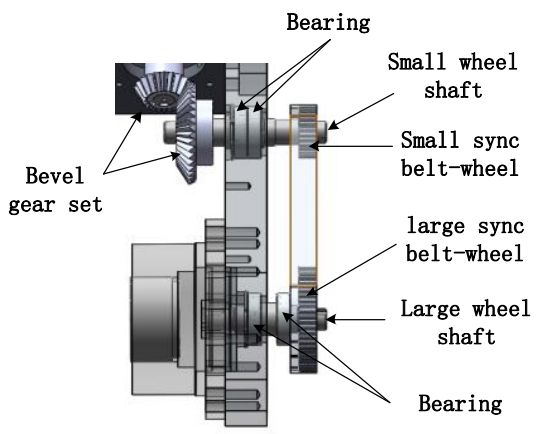

Figure9. Transimission relationship of Elbow joint

Table 1. Calculation of output torque and speed

\begin{tabular}{|c|c|c|c|}
\hline Motion & $\begin{array}{c}\text { Reducer } \\
\text { Ratio }\end{array}$ & $\begin{array}{c}\text { Torque } \\
\text { (Nm) }\end{array}$ & $\begin{array}{c}\text { Speed } \\
\text { (RPM) }\end{array}$ \\
\hline Shoulder joint & 600 & 192 & 5 \\
\hline Rotation joint & 100 & 32 & 30 \\
\hline Elbow joint & 400 & 128 & 7.5 \\
\hline
\end{tabular}

\section{Conclusion and Future Work}

Aging society brings a lot of problems, a large number of patients with hemiplegia need rehabilitation. Based on the analysis of the advantages and disadvantages of the domestic and foreign rehabilitation robot, from the medical point of view, analyze the physiological structure and movement characteristics of the human body, a novel multifunctional rehabilitation robot is designed . Compared with the existing rehabilitation robot, the biggest difference is that the design philosophy of the multi-functional support robot is based on the therapist in the patient rehabilitation 
training process of treatment. It is a high degree of intelligence, not limited to the inherent training methods, according to the patient's condition to develop specialized training programs .

As a future work, the mechanical structure of the multi-functional support rehabilitation robot will be further optimized. Further, we will build the robot control system and information detection system, information detection platform and quantitative evaluation system will be the focus of our work. In addition, in-depth cooperation with the hospital, clinical trials of hemiplegic patients to verify the effectiveness of rehabilitation robot system .

\section{Acknowledgement}

The authors are thankful to the financial support from Science and Technology Foundation of Guangdong (No.2016A020220001), Returned overseas business Foundation of Shenzhen (No. 2016001), Technology ResearchFoundation of Basic Research Project of Shenzhen (No. JCYJ20160428110654601 and JCYJ20160428110354308), Research Foundation of Health and Family Planning Commission of Shenzhen Municipality (No.201601054).

\section{References}

[1] Aging population development trend forecasting research reports of China. Members of the National Council on Aging office .2006.

[2] State Department China Aging Development "Twelve Five" plan, 2011.9.17: 2.

[3] Jishu Rong Practical hemiplegia rehabilitation technical illustration. People's Medical Publishing House 2005

[4] Carmen E. C., Mullens C. H., Brown D.A. Maximum walking speeds obtained using treadmill and overground robot system in persons with post-stroke hemiplegia [J]. Journal of NeuroEngineering \& Rehabilitation, 2012, 9(1): 80-93.

[5] Delussu A.S., Morone G., IosaM., Bragoni M., Traballesi M., Paolucci S. Physiological responses and energy cost of walking on the Gait Trainer with and without body weight support in subacute stroke patients [J]. Journal of NeuroEngineering \& Rehabilitation, 2014, 11(1): 54-93.

[6] Craven C.T.D. Development and evaluation of rehabilitation technologies for early-stage spinal cord injury [D]. Glasgow: University of Glasgow, 2014

[7] Aach M., Cruciger O., Meindl R.C., Sczesny-Kaiser M., Höffken O., Tegenthoff M., Schwenkreis P., Sankai Y., Schildhauer T.A. Voluntary driven exoskeleton as a new tool for rehabilitation in chronic spinal cord injury: a pilot study[J]. Spine Journal, 2014, 14(12):2847-2853.

[8] Woldag H, Hummelshcim H. Evidence based physiothera-peutic concepts for improving arm and hand function in apoplexy patients. Neurol, 2002, 249: 518 -528.

[9] Zhang Xinhong. Apoplexy rehabilitation. Medicine and Philosophy, 2004, 25 (7): 29.

[10] Human dimensions of Chinese adults. GB/T10000-1988. 